Ambiente \& Água - An Interdisciplinary Journal of Applied Science
ISSN 1980-993X - doi:10.4136/1980-993X
www.ambi-agua.net
E-mail: ambi-agua@agro.unitau.br

\title{
Análisis jurídico sobre la calidad del servicio del agua en Buenos Aires
}

\author{
doi: $10.4136 / a m b i-a g u a .1228$
}

Received: 23 Oct 2013; Accepted: 11 Mar. 2014

\section{Clara María Minaverry}

\author{
Universidad de Buenos Aires, y Consejo Nacional de Investigaciones Científicas y Técnicas (CONICET), \\ Buenos Aires, Argentina \\ Instituto de Investigaciones Jurídicas y Sociales Ambrosio Lucas Gioja, Facultad de Derecho \\ cminaverry@derecho.uba.ar
}

\section{RESUMEN}

El presente trabajo tiene como objetivo general analizar la eficacia de una serie de casos jurisprudenciales y de normativa seleccionada, en relación con la protección de la calidad del servicio de agua potable en la ciudad de Buenos Aires. En primer lugar, se recopiló doctrina, normativa y jurisprudencia nacional, todos vinculados con el estado de situación de la calidad de dicho servicio. Desde el punto de vista metodológico se utilizó el método de observación documental, y los datos cualitativos a recoger fueron secundarios (doctrina, normativa y jurisprudencia). A tal fin se analizaron profundamente tres leading cases, los cuales podrían fijar lineamientos generales para futuros proyectos de leyes y para políticas públicas que fomenten un servicio más sustentable.

Palabras-clave: jurisprudencia, agua, Argentina.

\section{Análise jurídica sobre a qualidade do serviço de água potável em Buenos Aires}

\section{RESUMO}

O presente trabalho teve como objetivo geral analisar a eficácia e alguns casos de jurisprudência e da norma legal selecionada pertinente à qualidade do serviço de água potável na cidade de Buenos Aires. Em primeiro lugar, foi realizado um levantamento da doutrina, leis e jurisprudência nacional, aspectos vinculados à qualidade da água na região avaliada. Do ponto de vista metodológico foi utilizado o método da observação documental por meio de dados qualitativos secundários (doutrina, leis e jurisprudência). Com tal fim, foram analisados profundamente três casos chaves, que podem estabelecer linhas gerais para futuros projetos de leis e políticas públicas para fomentar um serviço mais sustentável.

Palavras-chave: jurisprudência, água, Argentina. 


\title{
Legal analysis about drinking water quality service in Buenos Aires
}

\begin{abstract}
The general purpose of this paper was to analyze the efficacy of selected case law and regulations regarding piped water service quality in Buenos Aires. We searched first for jurisprudence, regulations and case law relating to the quality of water service in Buenos Aires. We used the documentary observation method, and the qualitative data were categorized as secondary (jurisprudence, regulations and case law). We analyzed three leading cases, which might be useful to set general guidelines for future law projects and public policies in order to have a more sustainable service.
\end{abstract}

Keywords: case law, water, Argentina.

\section{INTRODUCCIÓN}

El 60 o el 70\% aproximadamente del cuerpo humano está compuesto por agua, y hoy en día no cabe duda que la misma es limitada y que debe ser especialmente cuidada y valorada (Minaverry, 2013b). La mayor cantidad de agua es salada (el 97,5\%), mientras que el resto corresponde a lagos, ríos, reservorios, recursos subterráneos, humedad del suelo, glaciares y nieves. Por eso, debe prestarse especial atención a la fuente del agua que consumimos todos los días, ya que forma parte de esa minoría que es dulce y que no tiene que ser sometida al proceso de desalinización, que supone altos costos económicos y contaminación del medioambiente.

Otra limitación vinculada con los recursos hídricos se relaciona con la distribución de los mismos en el territorio, con la cantidad y con la ubicación de la población mundial. En Sudamérica la situación es muy favorable, porque el balance existente entre la disponibilidad del agua y el porcentaje de la población es positivo. Actualmente, existen más reservas de agua que cantidad de personas demandando su uso, ya que en esta área habita solamente el $6 \%$ de la población que posee el $26 \%$ de los recursos hídricos mundiales.

Sin embargo, algunos estudios informan que "en Argentina millones de personas se abastecen de agua no apta para el consumo humano ya que el recurso subterráneo está contaminado; que los pozos no tienen adecuada profundidad o carecen de los requisitos técnicos idóneos para evitar la infiltración de contaminantes" (CELS et al., 2009).

En este orden de ideas, se detectó un déficit en los servicios de suministro de agua potable en la mayoría de los países de Latinoamérica, debido a que los niveles de inversión histórica habían estado por debajo de sus necesidades (Lentini, 2011). Esto fue consecuencia de un congelamiento de las tarifas durante muchos años, a pesar de que al comienzo de las privatizaciones se habían registrado varias inversiones en infraestructura si se lo compara con el período de la empresa pública (Alcazar et al., 2000).

Algunos organismos internacionales ya habían detectado ciertos incumplimientos en nuestra región, al afirmar que durante algunos años en diversas ciudades se encontraron niveles excesivos de nitrato en agua de red y por cuatro años consecutivos los estudios revelaron un aumento en la cantidad de pozos con arsénico (Transparency International, 2008).

Para poder alcanzar los niveles básicos de calidad se requiere que el servicio cumpla satisfactoriamente con todas las obligaciones legales existentes, y a su vez que los usuarios brinden una contraprestación razonable del servicio.

"El suministro de agua potable, y en especial los servicios de alcantarillado y tratamiento de aguas servidas, requieren más inversión de capital que otros servicios de utilidad pública: casi veinticinco veces los ingresos anuales" (Solanes, 2008). 
Debe destacarse la importancia que existe en realizar inversiones adecuadas y planificadas para mantener el nivel de la infraestructura requerida para brindar el servicio, y así mantener un alto nivel de calidad del agua (Rouse, 2007).

En este sentido "se ha calculado que alrededor del $65 \%$ de los costos totales para el servicio de agua corresponden al capital, en tanto que la vida útil de los activos es de 20 a 40 años" (Ferro e Lentini, 2012).

Entre éstos aparece la obligación de brindar una adecuada presión que es considerada como la media ponderada en diez metros de columna de agua por inmueble. A su vez, se exige un adecuado sistema de monitoreo.

La continuidad se refiere a que la prestación no puede ser interrumpida y entre otras cosas, se relaciona con la inembargabilidad de los bienes afectados por ser de dominio público, es decir por constituir el conjunto de bienes propiedad del Estado afectados por la ley al uso directo o indirecto de los habitantes (Prado, 1997).

Algunos doctrinarios afirman muy acertadamente que "ciertos estándares no son negociables: la calidad química y bacteriológica del servicio tiene parámetros legales de cumplimiento obligatorio por cuestiones sanitarias y de la preservación de la vida. La reducción de los costos para controlar la calidad implica que en un determinado plazo se pagará esto de manera mucho más cara" (Lentini, 2011).

\section{OBJETIVOS Y JUSTIFICACIÓN}

Objetivo general: Analizar la eficacia de los casos jurisprudenciales y de la normativa seleccionada, en relación con la protección de la calidad del servicio de agua potable en la ciudad de Buenos Aires.

Objetivos específicos:

a) Analizar si la jurisprudencia y la normativa seleccionadas poseen sanciones que sean disuasorias ante casos de incumplimiento normativo en el nivel de calidad del suministro de agua potable en la ciudad de Buenos Aires.

b) Analizar si la jurisprudencia y la normativa seleccionadas poseen un sistema de control de calidad que haya sido ejercido por el Estado en el suministro de agua potable en la ciudad de Buenos Aires.

La importancia del presente trabajo radica en que para el caso de Argentina, la situación de la disponibilidad hídrica superficial (dejando de lado las fuentes subterráneas y de los glaciares), cuenta con una gran variedad de fuentes de recursos hídricos, en especial si lo comparamos con otros países de la región como Chile.

Sin embargo, muchos de los recursos hídricos están siendo contaminados, como consecuencia de escasos o ineficientes controles estatales, falta de educación ambiental y de responsabilidad individual y colectiva, y esto repercute directamente en la calidad del servicio de agua potable (si las empresas y el Estado no intervienen eficientemente).

El ámbito espacial de esta investigación (ciudad de Buenos Aires) se nutre de manera directa del recurso del agua, ya que puede acceder fácilmente al Río de la Plata. Esto le da ventaja en relación a otras zonas del país, como es el caso de algunas áreas del norte y centro.

\section{MATERIALES Y MÉTODOS}

a) Fase exploratoria:

Se recopiló normativa y jurisprudencia nacional vinculadas con el estado de situación de la calidad del servicio del agua potable en Buenos Aires. Además se realizó una selección de 
obras doctrinarias y de aplicación empírica sobre la temática de la calidad del agua. A tal fin se utilizaron los siguientes buscadores jurídicos: Microjuris, Ecolex y Lexpro, y luego se fueron detectando los aspectos fundamentales de la doctrina, normativa y de los casos jurisprudenciales.

b) Fase descriptiva:

1- La información recogida en la etapa anterior fue clasificada y categorizada (utilizando los criterios de división territorial, jurisdiccional y doctrinario), a fin de facilitar su análisis en una etapa posterior.

2- Se realizó una descripción de "casos" en los que el núcleo duro de la cuestión fue la calidad del agua. De ello obtuvimos información relevante para arribar a algunas conclusiones generales.

c) Fase analítica:

1- El análisis de los documentos vinculantes y no vinculantes recogidos en la fase exploratoria tuvo por objeto detectar los principios y marcos legales que, debido a su reiteración constante y uniforme, fueron tomados en cuenta para enriquecer el marco teórico de esta investigación.

2- Se categorizaron los estudios de caso publicados en revistas o por organismos nacionales e internacionales de reconocida trayectoria.

Desde el punto de vista metodológico, se utilizó el método de observación documental, el cual consiste en obtener información mediante la percepción selectiva, ilustrada e interpretativa de un fenómeno determinado. Dentro de las posibles modalidades se implementó la "observación directa", ya que los datos se recogieron directamente de los fenómenos percibidos mediante registros sistematizados con la recolección.

Asimismo se utilizaron los métodos analítico y comparativo de datos, siendo que se comparó legislación y jurisprudencia de distintas jurisdicciones de Argentina.

Los datos cualitativos recogidos fueron secundarios (análisis de registros escritos, tales como doctrina, jurisprudencia, y legislación).

\section{RESULTADOS Y DISCUSIÓN}

\section{1. Estado de situación del control de calidad del servicio de agua en Buenos Aires}

La actual empresa concesionaria que provee el servicio en Buenos Aires desde 2006 (Agua y Saneamientos Argentinos S.A) posee dos clases de controles de la calidad del agua:

a) Directos: Cuando los inspectores se dirigen a la industria y donde la Administración Pública es responsable directa del control.

b) Indirectos: Se mide a través de indicadores de cuencas. Su función principal es la de monitorear el manejo de los planes y acciones destinados a la gestión de una cuenca hídrica (Arg Cap-Net, 2013).

Ambos se definen conforme a lo establecido en la ley nacional 26.221 (Argentina, 2007) (Anexo A), donde se describen los niveles de calidad del agua exigidos para el consumo.

Se han intensificado los controles ambientales a partir de la firma de un convenio con la Autoridad de la Cuenca Matanza Riachuelo (ACUMAR), tal es el caso del control de cianuros. En caso de detectarse esta sustancia en el agua se ordena de inmediato el corte de las actividades de las industrias de la zona.

Además la empresa implementó lo siguiente:

a) Aplicación de estrictos controles durante las 24 horas, los 365 días del año.

b) Los controles de calidad que se realizan al agua abarcan todas las etapas del proceso. 
c) Las normas de calidad del agua que debe cumplir la empresa incluyen 58 parámetros definidos por el Marco Regulatorio, los cuales se basan en las exigencias del Código Alimentario Argentino, y de las principales guías y normas internacionales de referencia en la temática (Organización Mundial de la Salud (OMS).

d) El Laboratorio Central se encuentra equipado con tecnología de punta e instrumental de última generación, y con personal altamente capacitado (AYSA, 2013).

Las muestras de agua se toman en más de cuatrocientos puntos fijos de las redes distribuidoras, y se focaliza en los siguientes parámetros (turbiedad, análisis bacteriológicos, determinaciones biológicas, parásitos y otros patógenos), establecidos en la normativa que se analizará más adelante (AYSA, 2013). Se ha comenzado a trabajar interdisciplinariamente y conjuntamente entre la empresa, el Instituto Nacional del Agua (INA) y Redes de Laboratorio.

\subsection{Análisis sobre la normativa vinculada con la calidad del servicio del agua}

A continuación se describirá la normativa sobre calidad del agua, que es aplicable a los municipios de la Provincia de Buenos Aires. Las mismas han sido divididas en dos áreas:

a) Normas correspondientes al período concesionado a empresas privadas3:

Decreto Provincial 878/2003, capítulo V (Buenos Aires, 1996).

b) Normas correspondientes al período de provisión estatal del servicio:

Ley 26.221 de 2007, Anexo A y capítulo II punto 6.

De acuerdo a la normativa anterior, puede afirmarse que existe un solapamiento legal entre la ley nacional 26.221 de 2007 y el decreto provincial 878/2003, ya que ambos están vigentes para el ámbito de la Provincia de Buenos Aires.

En el artículo 8 inciso j) del decreto 878/2003 se incluyeron las definiciones aplicables a la norma, y allí se estableció que dicha Comisión será la autoridad que determinará las características que debe tener el agua para ser considerada potable.

La categoría de agua corriente que está prevista para consumo humano, no cuenta con ningún parámetro legal de calidad exigible a las empresas que proveen la misma.

Además, este decreto resulta paradigmático, ya que ha creado dos categorías de agua que están permitidas para el consumo humano pero que poseen características diferentes:

a) "Agua Potable: Agua que cumple con todos y cada uno de los límites impuestos por la Comisión Permanente de Normas de Potabilidad y Calidad de Vertido de Efluentes Líquidos y Subproductos.

b) Agua Corriente para el consumo humano e higiene: Agua que no cumple con algunos de los límites impuestos por la Comisión Permanente de Normas de Potabilidad y Calidad de Vertido de Efluentes Líquidos y Subproductos, pero cuya ingesta puede ser autorizada por períodos limitados"

En el inciso b) el término "algunos" aplicado a los límites de calidad del agua no ha sido definido y por lo tanto existe una laguna jurídica.

Además se establece que el agua corriente puede ser consumida por "períodos limitados", que tampoco se han definido y que pone en peligro la salud de los usuarios (cuya responsabilidad corresponde a la Administración Pública).

Luego, el instrumento de vinculación firmado entre Aysa S.A. y el Estado Nacional solicita que se cumpla con el marco regulatorio y con las normas que se vinculan con la calidad del agua potable (pero no explican a cuáles se refieren pudiendo detectarse una laguna jurídica). 


\subsection{Casos jurisprudenciales vinculados con el nivel de cumplimiento legal de la calidad del servicio del agua}

\subsubsection{El caso "Municipalidad de Berazategui contra Aguas Argentinas S.A. sobre ordinario"}

En este caso que tramitó ante la Cámara Federal de Apelaciones de La Plata en 2003, y se cuestionó la falta de inversión en el tratamiento de efluentes cloacales en áreas de tomas de agua potable, y el incumplimiento en el monitoreo de la calidad del agua.

Sin duda que aquí el bien jurídico protegido es el agua potable, ya que su objetivo es que sea de óptima calidad de acuerdo a los parámetros de salud abalados internacionalmente.

Desde el punto de vista procesal, se llegó a una instancia de apelación porque el juez de grado no hizo lugar a la medida cautelar solicitada por la parte actora, alegando que no se encontraban cumplidos y acreditados los requisitos exigidos por la ley tales como la verosimilitud en el derecho y el peligro en la demora.

En la misma se demandó a la empresa prestataria del servicio de agua (Aguas Argentinas S.A.), solicitando que se la condene para que cese de inmediato con la contaminación de las aguas del Río de la Plata, repare los daños ambientales e indemnice los daños civiles causados.

La actora amplió su pretensión contra el Estado Nacional, para que ordene la ejecución de un plan para la construcción de una planta de tratamiento de los efluentes cloacales que se vertían en las aguas del Río de la Plata.

En este caso no se mencionaron los principios de precaución ni de prevención, porque el daño ya se había producido, lo cual se justificó en el apartado 12 del fallo:

"Esta situación, además, pone en riesgo de contaminación a las tomas de agua potable situadas sobre la costa del Río de la Plata bajo condiciones meteorológicas desfavorables, como así también produce la degradación de la línea de costa en el entorno de vertido."

A esto hay que sumarle la situación denunciada por el Ente Regulador del Agua (ETOSS) por haberse detectado descargas de mercurio en el Río de la Plata (considerando 13).

Uno de los principios ambientales que se encuentran mencionados en la sentencia es el de "contaminador - pagador", que implica que los responsables de la contaminación o degradación deben soportar gastos necesarios para prevenir o corregir el deterioro ambiental, tratándose los "costos sociales" que antes no se incluían en estos cálculos.

Las fuentes del derecho que se han incorporado en este caso pertenecen al ámbito nacional y al internacional.

Resulta novedoso que se haya citado el caso "Municipalidad de Magdalena contra Shell CAPSA y otros sobre disposición de residuos peligrosos" de 2003, afirmando que la acción contaminante resulta ilícita por contrariar a las normas de la Constitución, a los tratados internacionales y a las leyes nacionales y genera prioritariamente la obligación de recomponer el medio ambiente.

Se ha reconocido expresamente que la demandada no ha cumplido legalmente con ciertos aspectos claves vinculados con los niveles de inversión de infraestructura:

"Aguas Argentinas no realizó las nuevas obras ni adelantó inversiones y, a menos de dos años del contrato, formuló una propuesta de renegociación [...] al tercer año de la concesión las inversiones no realizadas ya pasaban de los 300 millones y en los dos años siguientes el incumplimiento se agravó, acercándose al cumplirse el primer quinquenio a la mitad de lo comprometido."

La parte demandada sostuvo que se había cumplido con las normas de calidad vigentes, y que en el mismo no se exigió la implementación de inversiones como es el aumento de la 
capacidad de producción y la potabilización del agua, sobre todo en relación con la expansión del servicio de agua y en el sistema de saneamiento.

Podemos destacar que las lagunas normativas existentes en esta área, representan un aspecto fundamental que debería ser tenido en cuenta en un futuro inmediato.

Este caso también ha servido para subsanar lo anterior, ya que se estableció que a pesar de que la empresa ha cumplido con dicha exigencia normativa, esto no resulta suficiente como para eximir de responsabilidad a la misma.

Una de las temáticas del fallo, es que la actora acertadamente sostuvo lo siguiente:

"Se advierte una clara responsabilidad del Estado en el ejercicio, paradójicamente, de su poder reglamentario, tanto en su legítima acción como por sus injustificadas omisiones en el marco de la renegociación del contrato de concesión."

Al tratarse de un servicio público básico para la vida, se consideró como responsable al Estado (en todos los casos), dándole un lugar destacado en relación con la empresa.

Otro de los aspectos que cuestionó la parte demandada es que había solicitado una renegociación de su contrato, alegando lo siguiente:

"Los documentos con los que la propia empresa propuso la renegociación planteaban que la regulación exigió con demasiada rigidez el cumplimiento de un plan cuyas metas eran demasiado "ambiciosas" obedeciendo a las necesidades licitatorias".

Esta problemática también podría haber sido evitada si hubiese existido normativa con exigencias más estrictas, respecto del nivel de compromiso legal de las empresas que suministran el servicio del agua. Por tal insuficiencia, se produjo en varias ocasiones renegociaciones de las condiciones del contrato de concesión por parte de la empresa, lo cual se encuentra dentro del ámbito de legalidad (pacta sunt servanda).

La sentencia del tribunal de segunda instancia fue favorable a la actora al reconocerse la falta de inversión en infraestructura y en sistemas de control de calidad.

En la misma se ordenó a la empresa de agua que adopte las medidas necesarias para que en el transcurso de los próximos 18 meses, realice las obras tendientes a la construcción y puesta en marcha de la Planta Depuradora de líquidos cloacales ubicada en Berazategui, como la limpieza y prolongación del emisario cloacal existente en dicha localidad.

Ambos debían presentar mensualmente ante el Tribunal un informe sobre el avance de las obras, por lo que se ha contemplado una instancia posterior de seguimiento que resulta ser muy favorable. Se ha determinado una responsabilidad concurrente de la empresa de aguas y del Estado Nacional, lo cual resulta innovador ya que la mayoría de los casos jurisprudenciales analizados sobre esta temática reconocen alguna clase de responsabilidad con mayor implicancia hacia alguno de los demandados.

\subsubsection{El caso "Biondo Esteban contra Secretaría de Recursos Naturales y Ambiente Humano - Poder Ejecutivo Nacional sobre amparo"}

Un Concejal del Partido de Berazategui (Provincia de Buenos Aires, Argentina) promovió una acción de amparo contra la ex Secretaría de Recurso Naturales y Ambiente Humano (actual Secretaría de Ambiente y Desarrollo Sustentable de la Nación) en 1999, fundamentado en la afectación de sus derechos constitucionales de vivir en un ambiente sano y equilibrado y a la salud (artículos 41, 43 y 31 de la Constitución Nacional), y también por la grave contaminación que sufría el Río de la Plata.

En su recurso alegó que el mismo era una fuente de abastecimiento de agua potable, y al mismo tiempo cuerpo receptor de desagües cloacales e industriales sin tratamiento previo, provocando que el Río de la Plata vea agotada su capacidad de autodepuración.

Los demandados fueron responsabilizados por haber incumplido su deber de poder de policía, en tanto que debieron ejercer el control de la contaminación hídrica, y afirmaron que su obligación subsistía más allá de las funciones de fiscalización de organismos como el 
ETOSS (ya que éste se limitó al cumplimiento del contrato de concesión de Aguas Argentinas S.A). Aquí se reconocieron las limitaciones estructurales y de funcionamiento que registran estos organismos de control.

A los fines de proteger la calidad del agua potable, el actor solicitó que la ex Secretaría de Recursos Naturales y Ambiente Humano defina lo siguiente:

- "Un programa de control efectivo de los desechos industriales que llegaran directa o indirectamente al Río de la Plata (en especial los generados por las industrias).

- Un sistema de monitoreo permanente de la calidad de dichos efluentes.

- La reubicación de las tomas de agua del concesionario en el Río de la Plata para su potabilización.

- El cumplimiento de los plazos para la construcción de una planta de tratamiento de desechos cloacales e industriales de las tres colectoras máximas que tienen su desaguadero en Berazategui."

La sentencia de primera instancia rechazó la demanda de la actora basándose en cuestiones de forma pero no de fondo, a pesar de encontrarse en juego derechos constitucionales (principalmente los artículos 41, 43 y 31 ).

El juez consideró que no se había cumplido con los requisitos de admisibilidad de la acción de amparo, porque existían otros remedios legales para resolver la cuestión (pero nunca se especificaron cuáles eran).

La demandada ya había presentado el informe solicitado en el artículo 8 de la ley nacional 16.986 de acción de amparo de 1966, alegando que se encontraban ante la ausencia de un caso judicial y de un acto administrativo concreto que afecte los derechos constitucionales cuestionados.

Además sostuvo que se requería mayor amplitud probatoria, ya que se estaban sometiendo a revisión cuestiones propias del poder administrador, por lo que este procedimiento no era considerado como el más idóneo para la resolución de una problemática con complejidad jurídica.

La Cámara de Apelaciones revocó la sentencia de primera instancia y atendió parcialmente las peticiones de la actora, y resolvió requerir informes de los últimos cinco años a todos los demandados en un plazo de noventa días, basándose en la ley general del ambiente 25.675 de 2002 que otorga dicha potestad.

A su vez, exigió el cumplimiento del derecho de acceso a la información (principio fundamental del Derecho Ambiental), y de disponer de todas las medidas necesarias para ordenar o probar hechos que generan un daño a intereses colectivos (en este caso el ambiente).

El fallo de segunda instancia judicial ha sido contrario a lo resuelto en la primera, pero ha mantenido un adecuado criterio lógico.

En este caso se pudo vislumbrar también que en el informe del ETOSS se había aprobado un plan de saneamiento integral, que estaba incorporado en el contrato de concesión pero que estaba sujeto a su aprobación definitiva. Cabe destacar que en el ámbito del Derecho Ambiental la dilación del tiempo genera un mayor daño al medioambiente, y que en muchos casos el mismo puede ser irreversible.

\subsubsection{El caso "Conde, Alberto José Luis y otro contra Aguas Bonaerenses S.A. sobre amparo"}

Un grupo de ciudadanos de la localidad de Lincoln, Provincia de Buenos Aires, interpuso una demanda contra dicha empresa por recibir en sus hogares agua con un valor de arsénico que excedía lo recomendado por la Organización Mundial de la Salud.

La Corte Suprema de Justicia de la Provincia de Buenos Aires exigió a la empresa "Aguas Bonaerenses Sociedad Anónima (ABSA)", suministrar a los usuarios agua potable 
con niveles de arsénico inferiores a 0,01 miligramos por litro en el mes de noviembre de 2013. Debe destacarse que ese es el nivel impuesto en el Código Alimentario Nacional.

Esto ocurrió a partir de la reforma del año 2007, que reflejó los "valores guías" establecidos por la Organización Mundial de la Salud en el año 2004. Previamente, el nivel de tolerancia era más alto: permitía hasta 0,05 miligramos por litro. Sin embargo, el artículo 982 dispone que "Para aquellas regiones del país con suelos de alto contenido de arsénico, se establece un plazo de hasta 5 años para adecuarse al valor de $0,01 \mathrm{mg} / \mathrm{l}$ ". Ese plazo de cinco años venció en 2012 pero fue prorrogado en febrero del mismo año.

Sin embargo, la empresa de agua estableció su imposibilidad práctica de cumplir con este fallo judicial de inmediato, debido a la magnitud de la inversión que demandan las obras para remover el arsénico del agua.

\section{CONCLUSIONES}

A lo largo del trabajo se ha podido lograr el objetivo general que consistía en analizar la eficacia de los casos jurisprudenciales y de la normativa seleccionada, en relación con la protección de la calidad del servicio de agua potable en la ciudad de Buenos Aires. Se ha podido determinar que esta eficacia es parcial, ya que ninguno de las dos fuentes del Derecho analizadas brinda herramientas ni soluciones absolutas para resolver la problemática de la protección de la calidad del servicio de agua potable.

Luego respecto de los objetivos específicos, del análisis jurisprudencial surge que en los mismos las sanciones son más exigentes que en otros casos ambientales que se han resuelto a nivel nacional (Buenos Aires, 2008). A su vez, cuando se imponen sanciones, en la mayoría de los casos los estándares ambientales y los montos de las multas se encuentran desactualizados.

En los fallos judiciales seleccionados se le exigió al demandado una "obligación de hacer", que resulta fundamental para asegurar el acceso a un servicio de agua de buena calidad, mientras que el nivel de control por parte de los organismos responsables fue evaluado como deficiente por parte de los tribunales correspondientes.

También se exigió un plan de monitoreo (muy exhaustivo), que implica una obligación adicional a los requerimientos solicitados al Estado (actuando de forma adecuada en relación al control público).

En síntesis, en dicha jurisprudencia se establecieron sanciones que pueden resultar disuasorias de incumplimiento normativo en el nivel de calidad del suministro de agua potable en la ciudad de Buenos Aires, y la existencia de un sistema de control de calidad que fue ejercido por el Estado en el suministro de agua potable en la ciudad de Buenos Aires.

Sin embargo, en algunos casos jurisprudenciales hemos detectado una escasa voluntad de trabajo conjunto e interdisciplinario en red entre los diferentes poderes y organismos públicos (Capaldo, 2011).

Luego, también respecto de los objetivos específicos, del análisis normativo surgió que las consecuencias jurídicas que surgen de la clasificación del agua para la Provincia de Buenos Aires, es que ambas pueden ser consumidas por seres humanos, a pesar de que poseen distintos estándares de calidad. Esto implica que en la Provincia de Buenos Aires las exigencias para las empresas que proveen el servicio de agua son inferiores a las de otras jurisdicciones (como es el caso de Buenos Aires que surge del punto 4.2).

Esto también indica que los controles públicos son más leves, y lo mismo ocurrirá con el nivel de sanciones por incumplimiento $\mathrm{u}$ omisiones normativas.

Sin embargo, el caso de los Partidos de la Provincia de Buenos Aires que actualmente se encuentran incluidos dentro de la concesión de la empresa Aysa S.A., resulta paradigmático ya que es el único ámbito del país donde coexisten simultáneamente la jurisdicción nacional, 
provincial y municipal. Esto genera que se superpongan normativas, y que dos o más organismos sean competentes para aplicar diferentes regulaciones y para controlar la calidad del servicio.

En la norma tampoco se explica qué parámetros de calidad no se estarían cumpliendo, evitándose así saber si esto resulta o no riesgoso para la salud humana.

En síntesis, en dicha normativa no se establecieron sanciones que sean disuasorias de incumplimientos normativos en el nivel de calidad del suministro de agua potable en la ciudad de Buenos Aires, ni un sistema de control de calidad que haya sido ejercido por el Estado en el suministro de agua potable en la ciudad de Buenos Aires.

\section{REFERENCIAS}

ALCAZAR, L.; ABDALA, M.; SHIRLEY, M. The Buenos Aires water concession, The World Bank Development Research Group, Regulation and Competition Policy, p. 21-22, 2000.

AGUA Y SANEANIMENTOS ARGENTINOS S. A. - AYSA. Web site. Disponible en: http://www.aysa.com.ar. Acceso en: 20 julio 2013.

ARGENTINA. Cámara Federal de Apelaciones de La Plata. Municipalidad de Berazategui contra Aguas Argentinas S.A. sobre ordinario. [S.l.]: [s.n.], 2003.

ARGENTINA. Cámara Federal de Apelaciones de La Plata. Biondo Esteban contra Secretaría de Recursos Naturales y Ambiente Humano - Poder Ejecutivo Nacional sobre amparo. [S.1.]: [s.n.], 1999.

ARGENTINA. Ley 26.221, promulgada en 28 febrero de 2007. Disponible en: http://infoleg.mecon.gov.ar/infolegInternet/anexos/125000-129999/125875/norma.htm.

Acceso en: 11 marzo 2014.

BUENOS AIRES (Provincia). Ley 11820, promulgada en 17 julio 1996. Disponible en: http://www.gob.gba.gov.ar/legislacion/legislacion/l-11820.html. Acceso en: 11 marzo 2014.

BUENOS AIRES (Provincia). Cámara de Apelación en lo Contencioso Administrativo de San Martín. Fundación Ecosur Ecológica Cultural y Educ. desde los pueblos del Sur contra Municipalidad de Vicente López y otro sobre amparo. [S.1.]: [s.n], 2008.

BUENOS AIRES (Provincia). Suprema Corte de Justicia de la Provincia de Buenos Aires. Conde, Alberto José Luis y otro contra Aguas Bonaerenses S.A. sobre amparo. [S.1.]:[s.n.], 2013.

CAPALDO, G. Gobernabilidad ambiental y eficacia del Derecho: dos magnitudes del desarrollo sustentable. In: CAPALDO, G. (Ed.). Gobernanza y manejo sustentable del agua. 1. ed. Buenos Aires: Mnemosyne, 2011. p. 39. (Sinergias Ambientales).

CENTRO DE ESTUDIOS LEGALES Y SOCIALES (CELS); ASOCIACIÓN CIVIL POR LA IGUALDAD Y LA JUSTICIA (ACIJ); CENTER ON HOUSING AND RIGHTS AND EVICTION (COHRE). El acceso a agua segura en el área metropolitana de Buenos Aires. Una obligación impostergable: programa servicios públicos. Buenos Aires, 2009. p. 14.

FERRO, G.; LENTINI, E. Infraestructura y equidad social: experiencias en agua potable, saneamiento y transporte urbano de pasajeros en América Latina. Santiago de Chile: División de Recursos Naturales e Infraestructura, 2012. p. 13. 
LENTINI, E. Servicios de agua potable y saneamiento: lecciones de experiencias relevantes Santiago de Chile: CEPAL, 2011. p. 39.

MINAVERRY, C. Análisis sobre el cumplimiento legal de las inversiones en el servicio público del agua en Buenos Aires, Argentina. Actualidad Jurídica Ambiental, v. 6, p. 1-15, 2013a.

MINAVERRY, C. El Derecho como herramienta para la erradicación de escenarios propicios para la corrupción en el servicio de agua. Tesis (Doctoral) - Facultad de Derecho, Universidad de Buenos Aires, Argentina, 2013b.

PRADO, J. Manual de instituciones de derecho público. Buenos Aires: Abeledo Perrot, 1997. p. 260.

RED ARGENTINA DE CAPACITACIÓN Y FORTALECIMIENTO EN GESTIÓN INTEGRADA DE LOS RECURSOS HÍDRICOS (Arg Cap-Net). Web site. Acceso: 25 mayo, 2013.

ROUSE, M. Institutional governance and regulation of water services. Londres: IWA, 2007. p. 39.

SOLANES, M. Descentralización de servicios de agua, eficiencia y acuerdos de protección a la inversión internacional. In: CONFERENCIA INTERNACIONAL SOBRE GESTIÓN DEL AGUA EN PAÍSES FEDERALES Y SEMEJANTES A LOS FEDERALES, 1., 2008, Zaragoza. Memorias... [S.1.: [s.n.], 2008. p. 491-517.

TRANSPARENCY INTERNATIONAL. Informe global de la corrupción 2008: corrupción en el sector del agua. Berlín, 2008. p. 22-40. 